\title{
Carbon and phosphorus relations in mycorrhizal and non-mycorrhizal pine seedlings
}

\author{
J.V.D. Rousseau and C.P.P. Reid \\ Department of Forestry, University of Florida, IFAS, Gainesville, FL, U.S.A.
}

\section{Introduction}

Several physiological and developmental responses to mycorrhizal fungus inoculation and applied phosphorus were examined to determine which responses were similar to enhanced phosphorus nutrition and which were more unique to the mycorrhizal association.

\section{Materials and Methods}

Pinus taeda L. seedlings, initially grown for 10 wk under low $P$ conditions, were subjected to a $2 \times 2$ factorial design of $2 P$ levels 10.1 and 10.0 $\mu \mathrm{g} / \mathrm{g}$ of P) with and without inoculation of Pisolithus tinctorius (Pers.) Coker and Couch. After an additional 7 wk, photosynthesis by all seedlings was measured followed by harvesting and determination of $P$ content and dry weight of foliage, stem and roots.

\section{Results}

Non-mycorrhizal seedlings grown under the high phosphorus regime (HP-N) had a $55 \%$ greater photosynthetic rate and
$150 \%$ greater biomass than did nonmycorrhizal seedlings grown under the low phosphorus regime (LP-N) (Fig. 1a, b). Mycorrhizal seedlings grown under the low $P$ regime (LP-M) also had a $75 \%$ greater photosynthetic rate, but less than $30 \%$ greater biomass than did the LP-N seedlings. Mycorrhizal seedlings grown under the high phosphorus regime (HP-M) showed no difference in photosynthetic rate over the HP-N seedlings. However, HP-M seedlings had $12 \%$ less biomass than HP-N seedlings.

The phosphorus content and foliar and fine-root $P$ concentrations were, respectively, 8, 4 and 2 times greater in HP-N than in LP-N seedlings (Fig. 1c, d, e). LP-M seedlings showed similar but less dramatic increases over the LP-N seedlings in $P$ content and foliar $P$ concentration. However, there was a substantial increase in the fine-root $P$ concentration of the LP-M over the LP-N seedlings, even exceeding levels of the HP-N seedlings.

For seedlings grown under high phosphorus conditions, inoculation with mycorrhizal fungus significantly increased the foliar $P$ concentration.

Mycorrhizal development, as measured by the percentage of short-roots infected, 

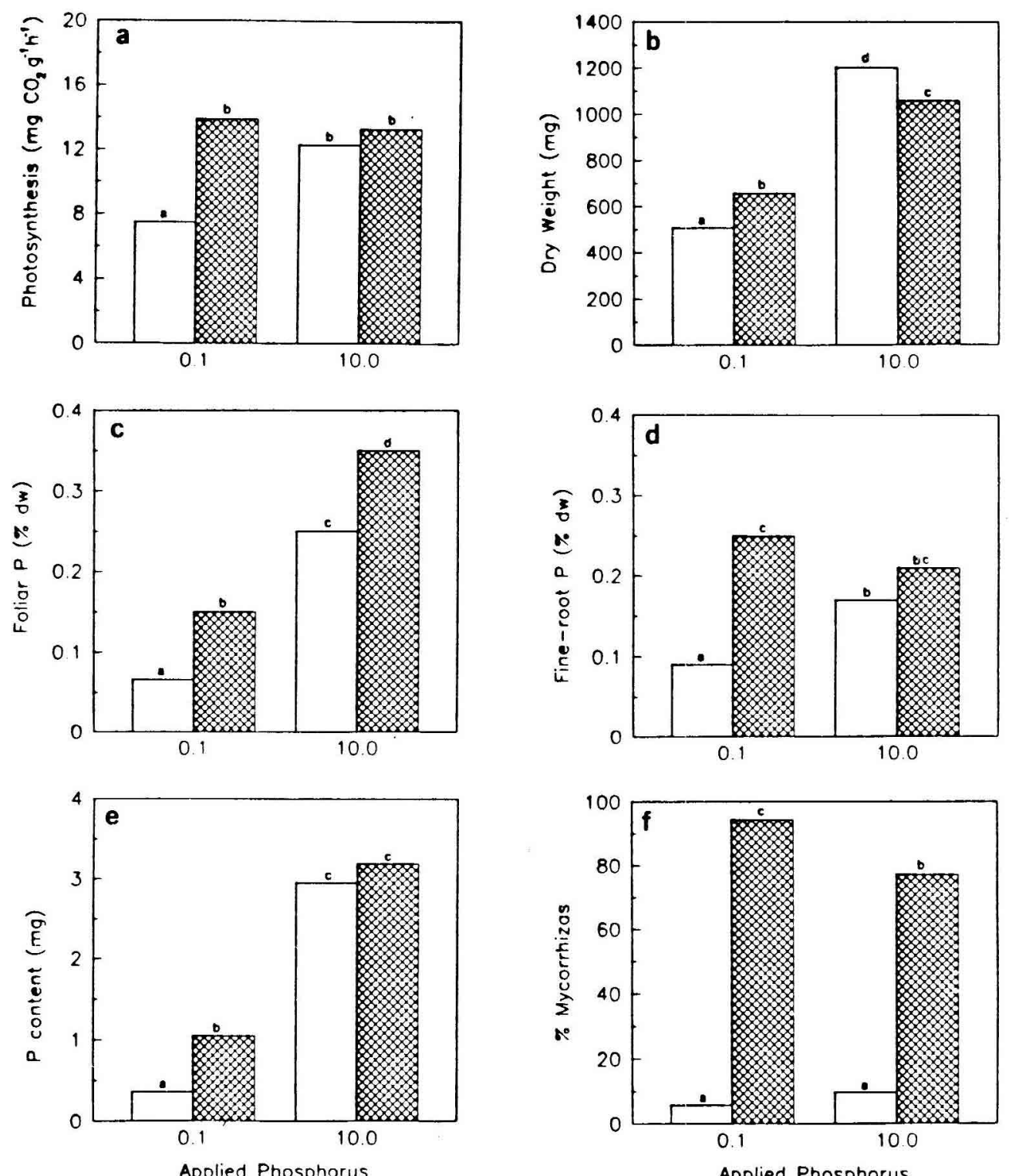

Fig. 1. Relationship among applied phosphorus levels $(0.1$ and $10.0 \mu \mathrm{g} / \mathrm{g}$ of P), mycorrhizal development (open bar: non-mycorrhizal; cross-hatched bar: mycorrhizal), and (a) photosynthesis, (b) dry weight, (c) foliar $P$, (d) fineroot $P$, (e) $P$ content of entire seedling and $(f)$ percentage of short-roots infected by $P$. tinctorius. Letters above bars indicate no significant difference in treatment means (ANOVA, Duncan's mean separation test, $P \leq 0.05$ ). 
was near $100 \%$ for the LP-M seedlings and almost $80 \%$ for the HP-M seedlings (Fig. 1f).

\section{Discussion and Conclusions}

Mycorrhizal development in seedlings grown under low $P$ conditions had a qualitative effect similar to adding phosphorus fertilizer to non-mycorrhizal plants for all parameters examined. In quantitative terms, adding high phosphorus had a far greater effect than inoculation, with two important exceptions, photosynthesis and fine-root phosphorus concentration. The high level of fine-root phosphorus concentration in mycorrhizal seedlings indicates that phosphorus was being preferentially stored in the mycorrhizal fungus, an idea originally suggested by the biochemical evidence of Harley and McCready (1952).
The similarity of the photosynthetic rates of LP-M plants with HP-N and HP-M plants, without a concomitant increase in dry matter, may suggest a substantial respiration cost of the mycorrhizas.

Mycorrhizal inoculation of seedlings grown with the high phosphorus fertilizer regime caused: 1) only a small increase in phosphorus content and concentration within the plant; 2) no change in photosynthetic rate; and 3) an actual $12 \%$ decrease in biomass. This biomass loss by mycorrhizal plants may represent increased respiration costs of the mycorrhizal root system.

\section{References}

Harley J.L. \& McCready C.C. (1952) The uptake of phosphate by excised roots of the beech. II. Distribution of phosphorus between host and fungus. New Phytol. 51, 343-348 